\title{
Softening of the equation of state of matter at large densities and temperatures: chiral symmetry restoration vs. quark deconfinement
}

\section{Luca Bonanno}

Dipartimento di Fisica, Università di Ferrara and INFN, Sez. Ferrara E-mail: bonannodfe.infn.it

\section{Alessandro Drago*}

Dipartimento di Fisica, Università di Ferrara and INFN, Sez. Ferrara E-mail: dragodfe.infn.it

\section{Andrea Lavagno}

Dipartimento di Fisica, Politecnico di Torino and INFN, Sez. Torino E-mail: andrea. lavagno@polito.it

\begin{abstract}
We discuss two models for describing the behavior of matter at large densities and intermediate temperatures. In both models a softening of the equation of state takes place due to the appearance of new degrees of freedom. The first is an hadronic model in which the softening is due to chiral symmetry restoration, while in the second model the softening is associated with the formation of clusters of quarks in the mixed phase. We show that both models allow a significant softening but, in the hadronic model the bulk modulus is mainly dependent on the density, while in the mixedphase model it also strongly depends on the temperature. We also show that the bulk modulus is not vanishing in the mixed phase due to the presence of two conserved charges, the baryon and the isospin one. Only in a small region of densities and temperatures the incompressibility becomes extremely small. Finally we compare our results with recent analysis of heavy ion collisions at intermediate energies.
\end{abstract}

Critical Point and Onset of Deconfinement - 4th International Workshop

July 9 - 13, 2007

Darmstadt, Germany

\footnotetext{
* Speaker.
} 


\section{Introduction.}

The behavior of matter at large densities and temperatures is still poorly known but, on general grounds, the appearance of new degrees of freedom is expected, leading to a softening of the equation of state (EOS). In a recent work [1] we studied two models, both allowing a softening of the EOS at large densities and/or temperatures. The first model is an hadronic model, based on a chirally symmetric EOS [, , 田 and the softening is due to partial restoration of the chiral symmetry. The second one is a mixed-phase model, where the softening is due to the formation of clusters of quarks, which are the precursors of deconfinement. These clusters are at first metastable and they stabilize only at larger densities.

The aim of our calculation is to compare how the softening takes place in the two models and finally to relate our results to recent analysis of the experimental data. This may be helpful also in prevision of future experiments planned e.g. at facility FAIR at GSI [5].

In our work we show that the behaviour of the incompressibility is qualitatively different in the two models: while in the chiral model the incompressibility depends strongly on the density and very weakly on the temperature (at least up to $T \sim 150 \mathrm{MeV}$ ), at the contrary in the mixed-phase model it also strongly depends on the temperature.

Finally, we compare our results with a recent experimental analysis [5], which suggests a progressive softening of the EOS, tested through HICs at energies ranging from $2 A \mathrm{GeV}$ up to $8 \mathrm{~A}$ $\mathrm{GeV}$. Since in HICs at intermediate energies not too large densities are reached, the mixed-phase model seems to provide a better description of the results of the experimental analysis.

\section{The Chiral-dilaton model.}

It is not trivial to develop chiral invariant models in which saturation properties of nuclei are well reproduced. It is well known that the attempt of using e.g. the sigma model to describe nuclear dynamics fails due to the impossibility to reproduce basic properties of nuclei [7]. More sophisticated approaches have been proposed in the literature, both within a SU(2) chiral symmetric models [ [8, 9] and also extending the symmetry to the strange sector [10, 11, 12]. Here we use the model introduced by the Minnesota group [2, 证. In that model chiral fields are present together with a dilaton field which reproduces at a mean field level the breaking of scale symmetry which takes place in QCD. In [2, 3, 4 it has been developed a formalism (which we adopt) allowing resummations beyond mean field approximation. This is important when studying a strongly nonperturbative problem as the restoration of chiral symmetry. The lagrangian of the model reads:

$$
\begin{aligned}
\mathscr{L} & =\frac{1}{2} \partial_{\mu} \sigma \partial^{\mu} \sigma+\frac{1}{2} \partial_{\mu} \pi \cdot \partial^{\mu} \pi+\frac{1}{2} \partial_{\mu} \phi \partial^{\mu} \phi-\frac{1}{4} \omega_{\mu v} \omega^{\mu v} \\
& -\frac{1}{4} B_{\mu \nu} \cdot B_{\mu v}+\frac{1}{2} G_{\omega \phi} \phi^{2} \omega_{\mu} \omega^{\mu}+\frac{1}{2} G_{b \phi} \phi^{2} b_{\mu} \cdot b^{\mu} \\
& +\left[\left(G_{4}\right)^{2} \omega_{\mu} \omega^{\mu}\right]^{2}-\mathscr{V} \\
& +\bar{N}\left[\gamma^{\mu}\left(i \partial_{\mu}-g_{\omega} \omega_{\mu}-\frac{1}{2} g_{\rho} b_{\mu} \cdot \tau\right)-g \sqrt{\sigma^{2}+\pi^{2}}\right] N
\end{aligned}
$$

where

$$
\mathscr{V}=B \phi^{4}\left(\ln \frac{\phi}{\phi_{0}}-\frac{1}{4}\right)-\frac{1}{2} B \delta \phi^{4} \ln \frac{\sigma^{2}+\pi^{2}}{\sigma_{0}^{2}}
$$




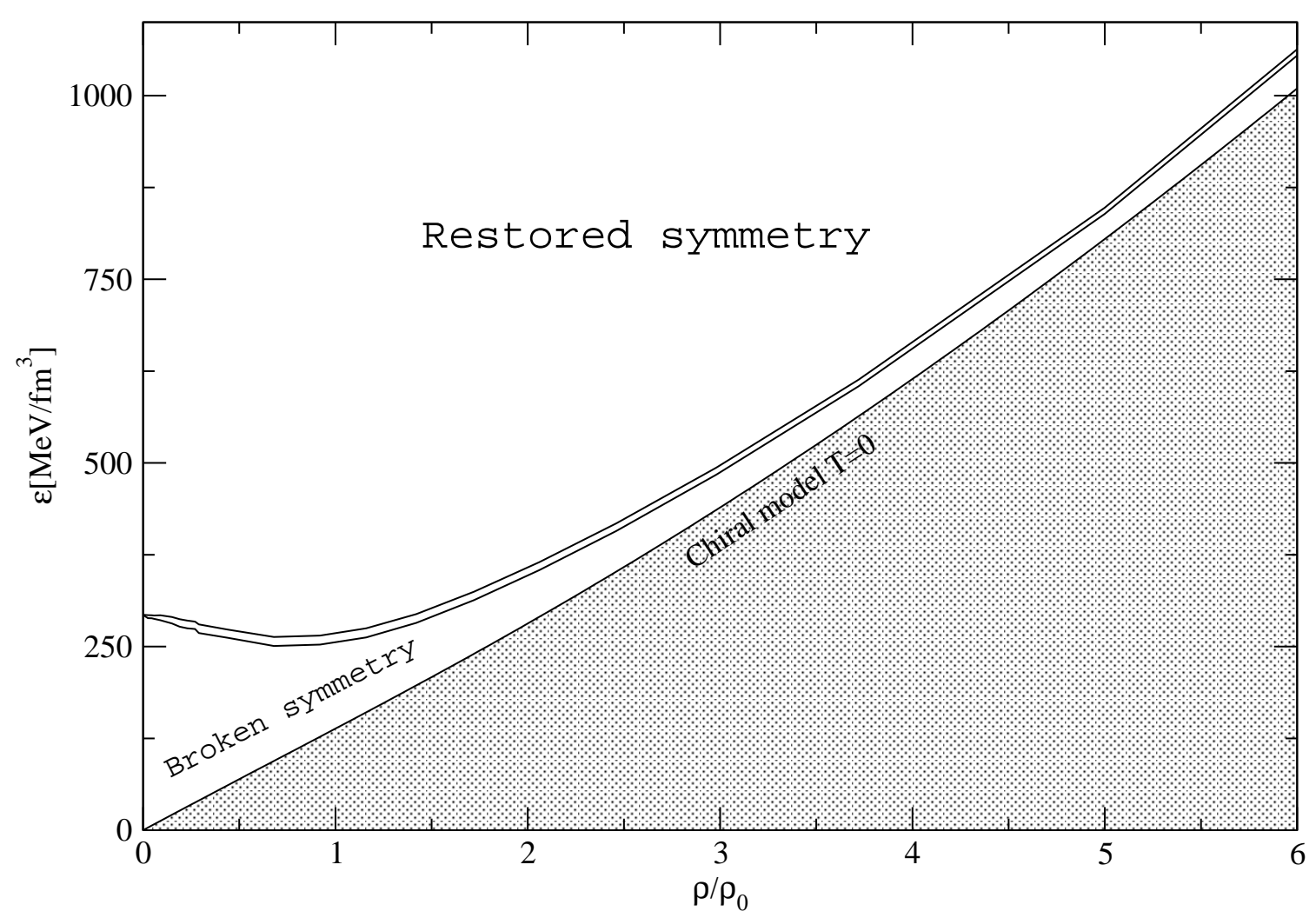

Figure 1: The figure shows the first order chiral transition, in the energy density vs baryon density plane, obtained using the chiral-dilaton model with $\varepsilon_{1}^{\prime}=0$ (which means that the lagrangian is exactly chirally invariant). Notice that, due to a first order chiral transition, the energy density has a slight discontinuity when the chiral symmetry is restored.

$$
\begin{aligned}
& +\frac{1}{2} B \delta \zeta^{2} \phi^{2}\left[\sigma^{2}+\pi^{2}-\frac{\phi^{2}}{2 \zeta^{2}}\right]-\frac{3}{4} \varepsilon_{1}^{\prime} \\
& -\frac{1}{4} \varepsilon_{1}^{\prime}\left(\frac{\phi}{\phi_{0}}\right)^{2}\left[\frac{4 \sigma}{\sigma_{0}}-2\left(\frac{\sigma^{2}+\pi^{2}}{\sigma_{0}^{2}}\right)-\left(\frac{\phi}{\phi_{0}}\right)^{2}\right] .
\end{aligned}
$$

Here $\sigma$ and $\pi$ are the chiral fields, $\phi$ the dilaton field, $\omega_{\mu}$ the vector meson field and $\mathbf{b}_{\mu}$ the vectorisovector meson field, introduced in order to study asymmetric nuclear matter. The field strength tensors are defined in the usual way $F_{\mu \nu}=\partial_{\mu} \omega_{v}-\partial_{\nu} \omega_{\mu}, \mathbf{B}_{\mu v}=\partial_{\mu} \mathbf{b}_{v}-\partial_{\nu} \mathbf{b}_{\mu}$. In the vacuum $\phi=\phi_{0}, \sigma=\sigma_{0}$ and $\pi=0$. The $\omega$ and $\rho$ vacuum masses are generated by their couplings with the dilaton field so that $m_{\omega}=G_{\omega \phi}^{1 / 2} \phi_{0}$ and $m_{\rho}=G_{\rho \phi}^{1 / 2} \phi_{0}$. Moreover $\zeta=\phi_{0} / \sigma_{0}$, B and $\delta$ are constants and $\varepsilon_{1}^{\prime}$ is a term that breaks explicitly the chiral invariance of the lagrangian. The potential $\mathscr{V}$ of eq. (2.2) is responsible for the scale symmetry breaking. The choice of such a potential comes from the necessity to reproduce the same divergence of the scale current as in QCD. In our calculation we use the parameters set of Ref.[ [7] which was able to reproduce nuclear spectroscopy and also gives, within this model, the smallest value for the incompressibility at saturation density, $K^{-1}=$ $322 \mathrm{MeV}$. Notice that this value is slightly larger than those traditionally used.

Using the model in the chiral limit $\left(\varepsilon_{1}^{\prime}=0\right)$, it is possible to obtain a first order chiral transition, as shown in Fig. 1. Notice that, due to the first order transition, the energy density has a slight 
discontinuity when the chiral symmetry is restoring. In the real case where the pion as a finite mass, the chiral symmetry is not restored, but there there is a crossover.

\section{The mixed hadron-quark phase.}

If the deconfinement transition at finite density is first order, a mixed phase can form and it is typically described using two separate EOSs, one for the hadronic and one for the quark phase. Concerning the hadronic phase we use a relativistic field theoretical model, the NL $\rho \delta$ [13], taking into account also a scalar-isovector interaction, which increases the symmetry energy only at large densities. Qualitatively similar results can be obtained using other hadronic models. For the quark phase we adopt an MIT bag like model. It is well known that, using the simplest version of the MIT bag model, if the bag pressure $B$ is fixed to reproduce the critical temperature computed in lattice QCD, then at moderate temperatures the deconfinement transition takes place at very large densities. On the other hand there are strong theoretical indications that at moderate and large densities (and not too large temperatures) diquark condensates can form, whose effect can be approximately taken into account by reducing the value of the bag constant. A phenomenological approach can therefore be based on a density dependent bag constant, as proposed in Refs.[14, 15]. We have adopted a parametrization of the form

$$
B_{\text {eff }}=B_{0}-[\Delta(\mu)]^{2} \mu^{2},
$$

where $\Delta(\mu)=\bar{\Delta} \exp \left[-\left(\mu-\mu_{0}\right)^{2} / a^{2}\right]$. Here $B_{0}{ }^{\frac{1}{4}}=215 \mathrm{MeV}, \bar{\Delta}=100 \mathrm{MeV}, \mu_{0}=300 \mathrm{MeV}$ and $a=300 \mathrm{MeV}$. One gluon exchange corrections are taken into account and we use $\alpha_{s}=0.35$. One constraint on the parameter values is that at $\mu=0$ the critical temperature is $\sim 170 \mathrm{MeV}$, as suggested by lattice calculations, while the other constraint is the requirement that the mixed phase starts forming at a density slightly exceeding $3 \rho_{0}$ for a temperature of the order of $90 \mathrm{MeV}$ (as also suggested e.g. by [16]). The choice of the parametrization is clearly inspired by the results of microscopical analysis on diquark condensate (for recent reviews see e.g. [17, 18]). On the other hand, the actual formation of a condensate and, even more, its specific type are still very uncertain ${ }^{1}$, what justifies a phenomenological approach as ours.

To describe the mixed phase we use the Gibbs formalism, which in Refs. [19, 20, 21] has been applied to systems where more than one conserved charge is present. In our work [1] we are studying the formation of a mixed phase in which both baryon and isospin charge are preserved 2 . The main result of this formalism is that, at variance with the so called Maxwell construction, the pressure is not constant in the mixed phase and therefore the incompressibility does not vanish. From the viewpoint of Ehrenfest's definition, the transition with two conserved charges is not of first, but of second order [20, 21].

An important issue concerns the effect of a surface tension at the interface between hadrons and quarks. The value of such a tension is poorly known, and in an MIT-bag-like model it is dominated by the effect of finite masses [22], in particular of the strange quark. On the other hand in the system

\footnotetext{
${ }^{1}$ The possible condensate would be build on $\mathrm{u}$ and d quarks. It is uncertain if such a condensate can exist at temperatures of several ten $\mathrm{MeV}$, if it can form in partially isospin asymmetric matter and if it produces a stable state.

${ }^{2}$ In Ref.[16] the same formalism has been adopted, but the conserved charges were the baryonic one and strangeness.
} 

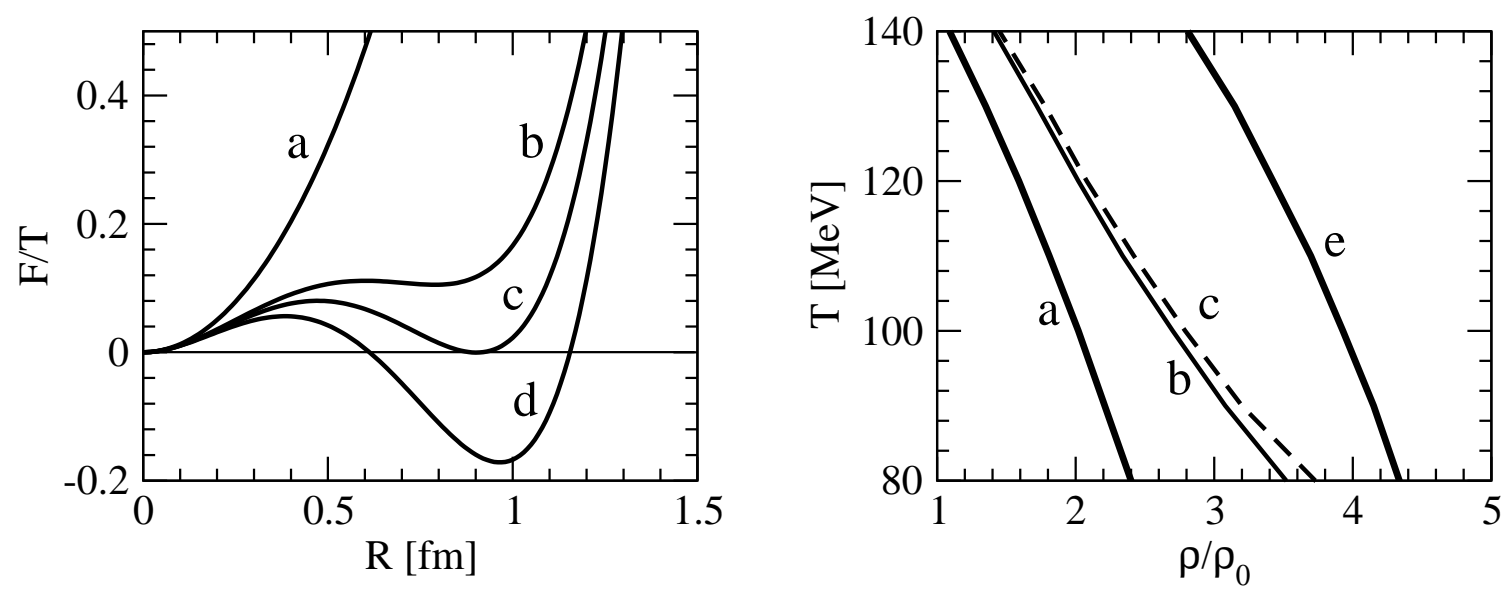

Figure 2: The upper panel represents the free energy of a bubble of quarks as a function of its radius R, while in the lower panel the temperature vs. the baryon density is shown. The $a$ and $e$ curves correspond to the critical densities delimiting the mixed phase in the absence of surface tension; the densities between $b$ and $c$ correspond to the formation of a metastable bubble. For densities larger than $c$ the mixed phase is stable (an example of the corresponding free energy is provided by the $d$ curve).

studied here strangeness plays a minor role, if any, and therefore the surface tension $\sigma$ should be rather small. In the analysis we have used $\sigma=10 \mathrm{MeV} / \mathrm{fm}^{2}$, but the results are qualitatively similar if a slightly larger value of $\sigma$ is used. In the upper panel of Fig. 2 we show the free energy for the nucleation of a bubble of quarks for various values of the density, computed using the formalism of Ref. [23]. One can notice that the density region in which quark bubble nucleation can really form shrinks respect to the case with $\sigma=0$. Moreover, there is an intermediate density region in which bubbles can form, but they are metastable. Only at larger densities a stable mixed phase can form. In our calculation this region is rather tiny, as shown in the lower panel of Fig. Z, but its size would increase for larger values of $\sigma$. This region of metastable quark bubbles is conceptually very interesting, because it provides a link with the so-called hadron-string-dynamics model [24]. In both descriptions, metastable matter is produced in an intermediate density window. In the string formation scenario that matter is made of unstable hadrons while in the scenario here presented it is made of (small) unstable bubbles of quarks.

\section{Results.}

We start by comparing in Fig. 3 the pressure computed using the models here discussed with the limits obtained from the analysis of HICs at intermediate energies [25]. It can be seen that the pressure computed using the chiral-dilaton model marginally exceeds the limit at low densities, due to the too large value of the incompressibility at saturation density. The effect of the partial restoration of chiral symmetry is clearly visible as a softening taking place at larger densities. Instead the introduction of a small fraction of strangeness to the Walecka GM3 model, does not produce a sizable softening of the EOS. ${ }^{3}$

\footnotetext{
${ }^{3}$ In HICs a small number of hyperons is generated through associated production. An estimate of the number of hyperons per participant can be obtained from the experimental ratio of the kaon yields per participant [26], which
} 


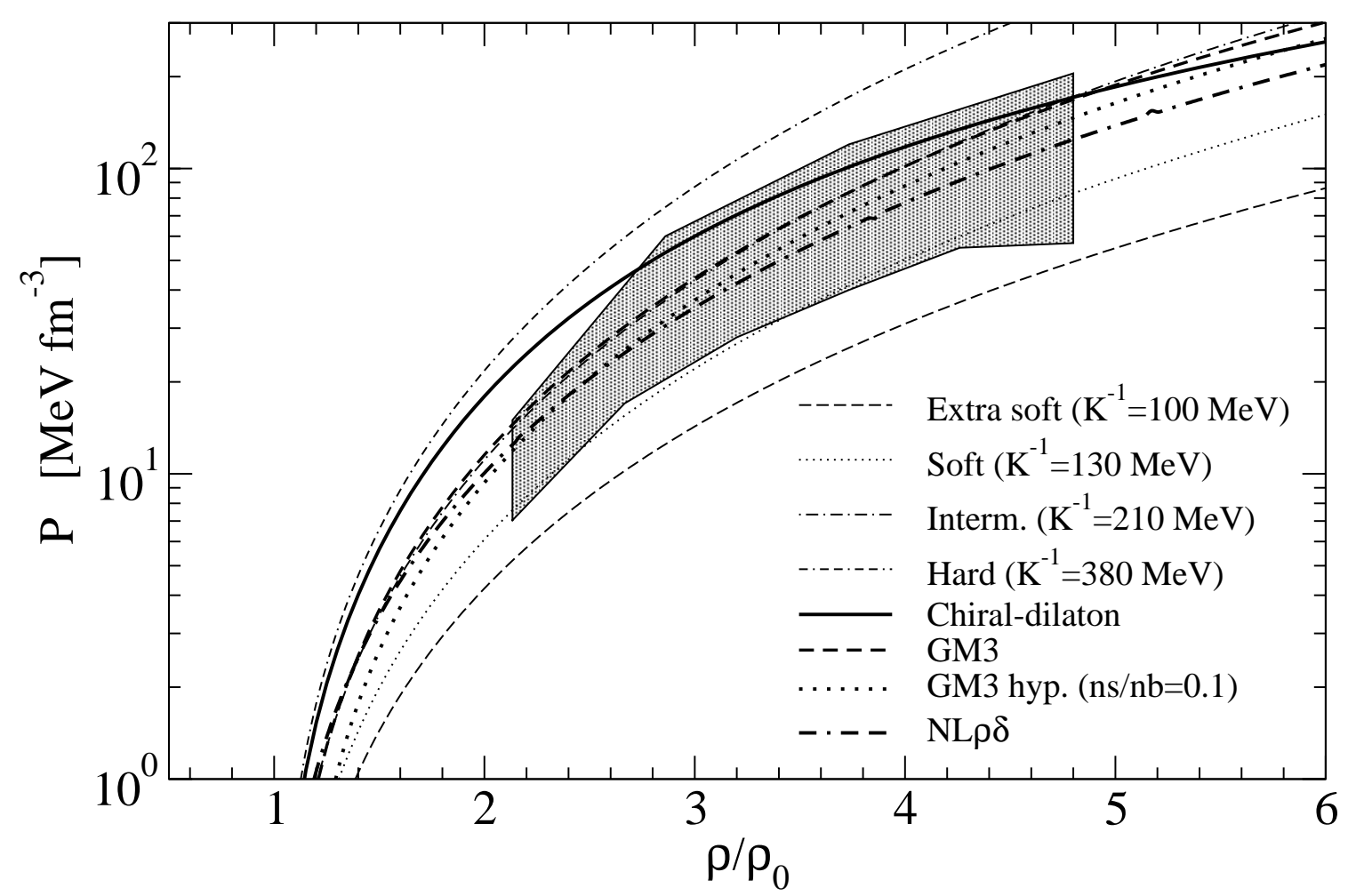

Figure 3: Pressure at $\mathrm{T}=0$ for symmetric nuclear matter, obtained using the chiral-dilaton model, the NL $\rho \delta$ model, the GM3 model and the set of simple parametric EOSs used in Ref. [6]. Also the pressure obtained using the GM3 with a little hereon fraction is shown.

In Fig. 7 we show the pressure over energy density ratio $p / \varepsilon$ as a function of $\varepsilon$ for different temperatures. Looking at the lower panel of Fig. $甘$, one notice that the ratio $p / \varepsilon$ starts to decrease at the beginning of the mixed-phase, reaching a minimum when pure quark matter is formed. This minimum is better known as the "softest point" [27], and it is expected to be visible at high energy HICs, as a signal of the deconfinement. From our calculations we can deduce that, for temperatures up to $120 \mathrm{MeV}$, which are reached at intermediate energies HICs, the signals associated with the softest point should be rather small.

We now compare the compressibility computed in the various models discussed above with the one estimated in [6]. In the following we always assume that $Z / A=0.4$. In the lower panels of Figs. 5 and 6 we plot the bulk modulus $B=\rho \partial P / \partial \rho$, respectively for the chiral dilaton model and for the mixed-phase model. Similarly, in the upper panels we show, as a function of the density and temperature, the value of the incompressibility parameter for which the bulk modulus of a parametric EOS has the same value of the bulk modulus computed in our models. This quantity, called $\widetilde{K}^{-1}$ is obtained by solving the equation:

$$
\left.\frac{\partial P_{\text {par }}(\tilde{K})}{\partial \rho}\right|_{\rho, T}=\left.\frac{\partial P_{\text {model }}}{\partial \rho}\right|_{\rho, T}
$$

indicates a strangeness fraction smaller than $10 \%$ Therefore in the analysis of the mixed phase we have neglected the strangeness content. 


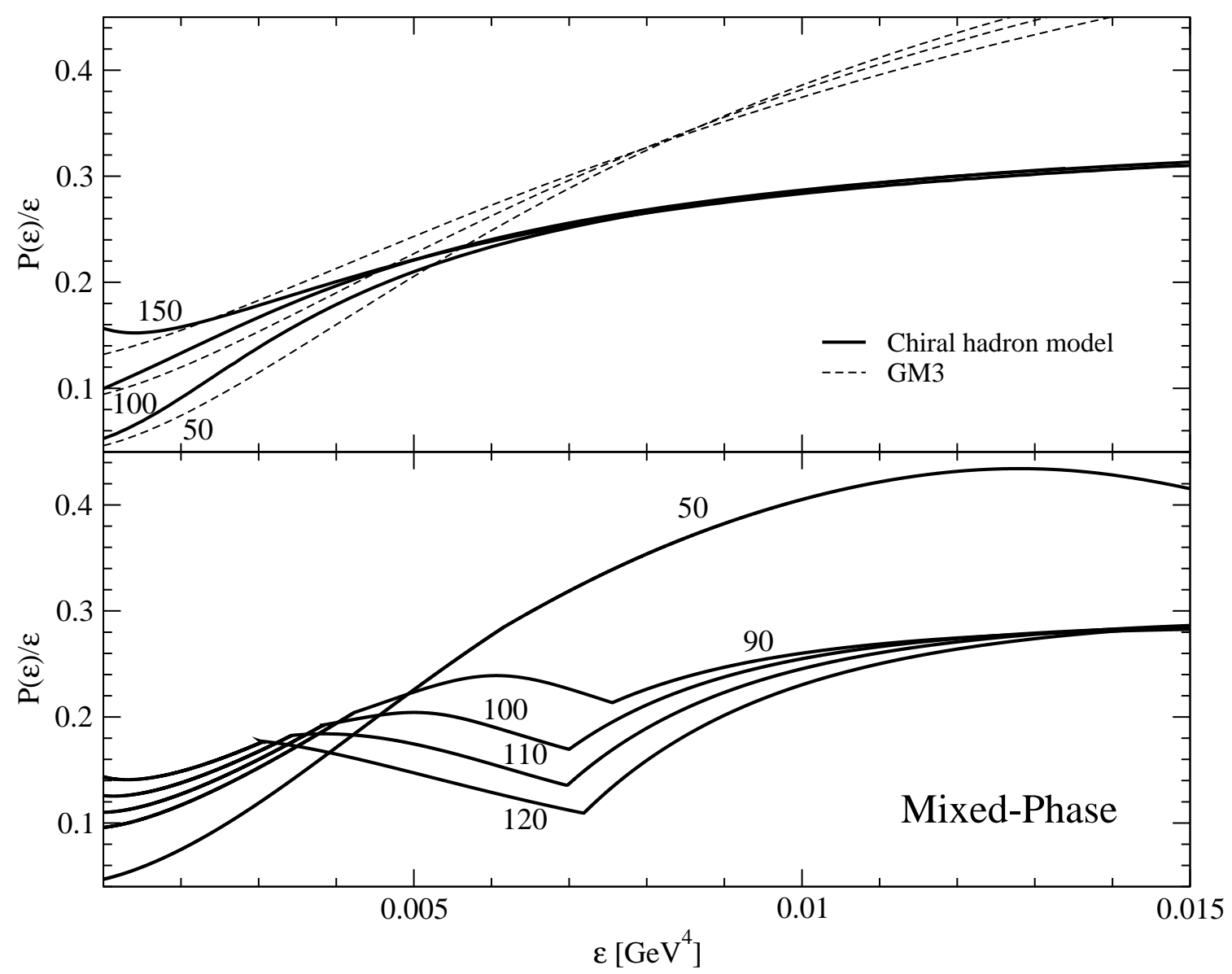

Figure 4: The figure shows the pressure over energy density ratio as a function of the energy density for different temperatures (the values of the temperatures in $\mathrm{MeV}$ are indicated by the numbers on the picture). The upper panel shows the results obtained using the chiral-dilaton and Walecka GM3 models, while the lower panel shows the results obtained using the mixed-phase model.

In this way we can directly compare with the analysis of [6] which explicitly indicates various values of the parameter $\widetilde{K}^{-1}$ as representative of the EOS tested at various energies. In the upper panel of Fig. 5 we show the $\widetilde{K}^{-1}$ parameter computed using the chiral-dilaton model and we see that it decreases significantly at large densities, while its dependence on the temperature is relevant only at small densities. By comparison, the GM3 parametrization provides a $\widetilde{K}^{-1}$ which is roughly constant at large densities.

In Fig. 6, we show that the incompressibility computed using the mixed-phase model remains rather large above the lower critical density and it becomes really small only approaching the upper critical density. It is extremely interesting to notice the strong dependence of the incompressibility on the temperature. For instance, at $\mathrm{T}=50 \mathrm{MeV}$ and $\rho=3 \rho_{0}$ we still obtain $\widetilde{K}^{-1} \sim 220 \mathrm{MeV}$, but at $\mathrm{T}=90 \mathrm{MeV}$ and $\rho=3.4 \rho_{0}$ we obtain $\widetilde{K}^{-1} \sim 170 \mathrm{MeV}$ and at $\mathrm{T}=100 \mathrm{MeV}$ and $\rho=3.6 \rho_{0}$ then $\widetilde{K}^{-1} \sim 120 \mathrm{MeV}$. The density-temperature region where incompressibility is extremely small is actually rather limited, because for $T \gtrsim 150 \mathrm{MeV}$ and $\rho \gtrsim 4.5 \rho_{0}$ the pure quark matter phase is reached, for which incompressibility is large again. Notice also that, while at lower temperatures 


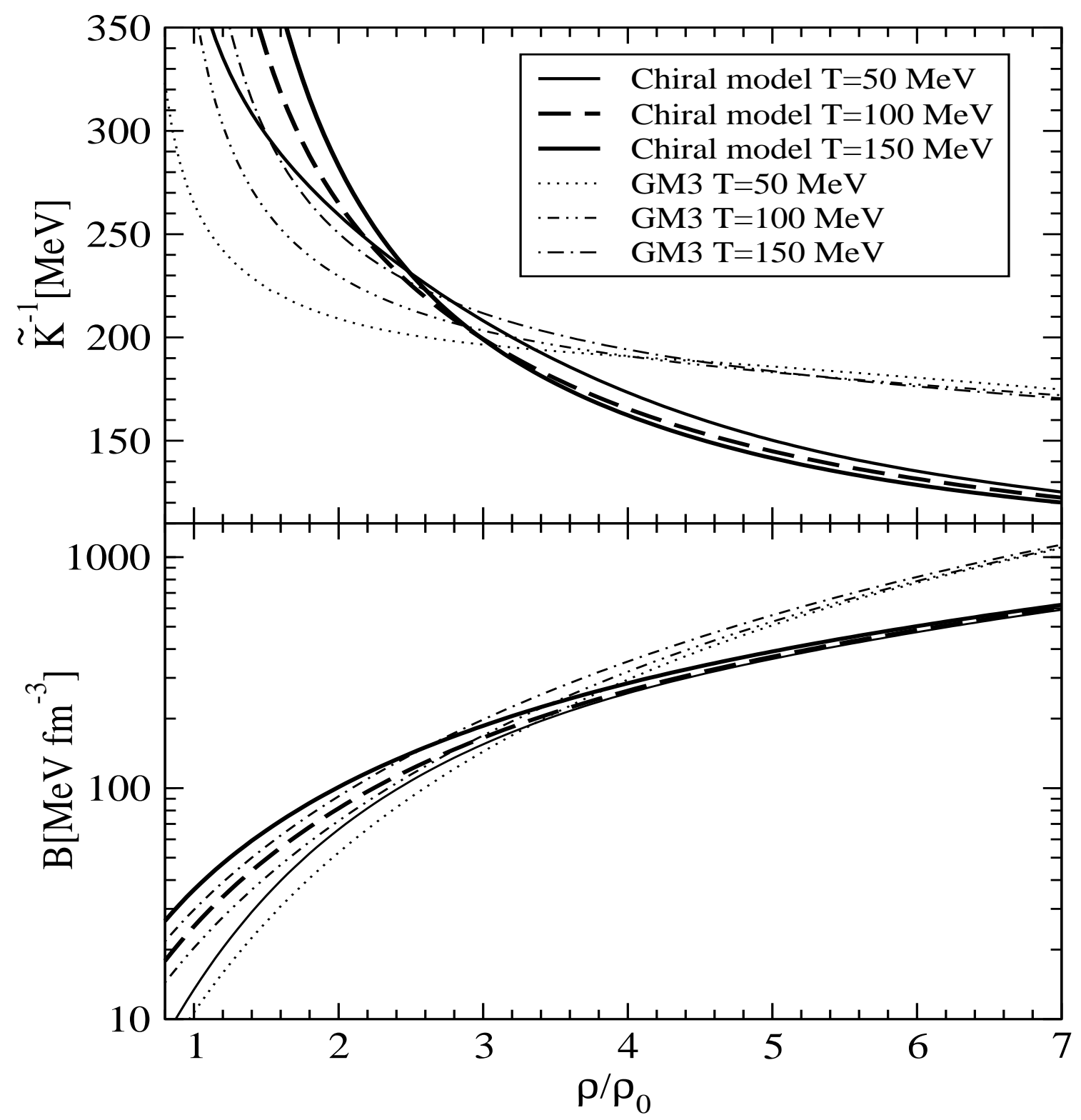

Figure 5: The upper and lower panels show respectively the parameter $\widetilde{K}^{-1}$ and the bulk modulus at different temperatures as a function of density for the chiral-dilaton model and the GM3 parametrization. Here we used $Z / A=0.4$

the values of the incompressibility is rather large, when T exceeds $\sim 120 \mathrm{MeV}$ the incompressibility is so small that, from practical purposes, the transition can be considered a first order.

A possible scenario based on the mixed-phase formation is the following. At $40 \mathrm{~A} \mathrm{GeV}$ the collapse of the elliptic flow has been interpreted as a signal of a first order phase transition to quarkgluon plasma [28, 29]. At lower energies, a mixed phase is produced, but due to the existence of two conserved charges the transition is of second order. The pressure is not constant and therefore dramatic manifestations of the formation of a mixed-phase are not expected. For instance, no wiggle in the behavior of the directed flow vs. rapidity is expected, since the appearance of a "third 


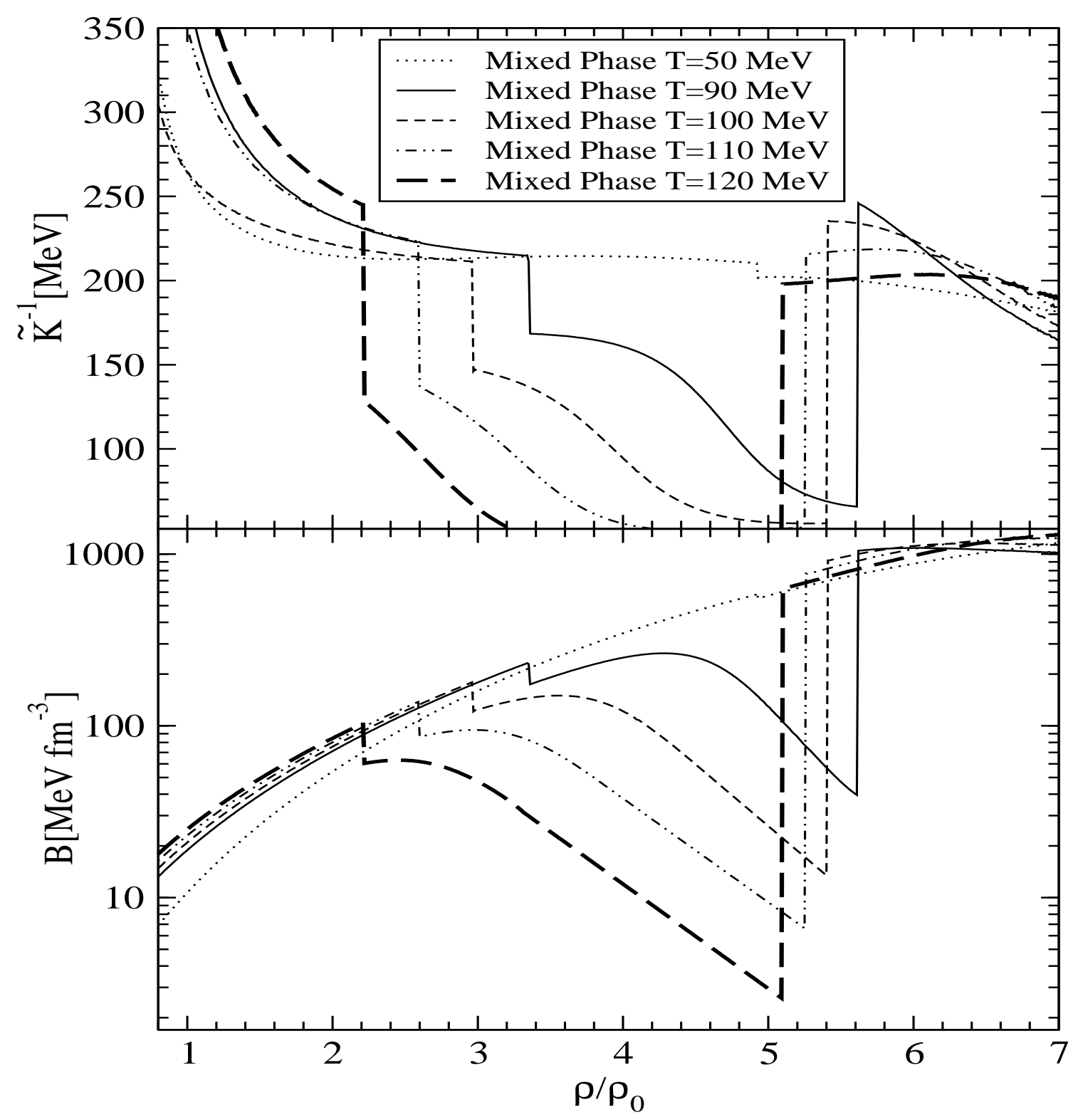

Figure 6: Same as in Fig. 5, but using the mixed-phase model.

flow component" [30] is strictly related to the existence of a first order transition. Only rather sophisticated analysis, as e.g. the extraction of the bulk modulus, can reveal the formation of a mixed phase. It will be interesting to test in future experiments if the oscillatory behavior of the directed flux, observed at $40 \mathrm{~A} \mathrm{GeV}$, does gradually take place at energies exceeding $\sim 10 \mathrm{~A} \mathrm{GeV}$ [5], due to a gradual shift from a second order to a "almost" first order phase transition, as suggested by our analysis.

In conclusion, a significant softening of the EOS can be obtained either via chiral symmetry restoration, if large densities are reached, or via the formation of a mixed phase of quarks and hadrons. The gradual reduction of the bulk modulus discussed in our analysis applies to all situations in which the mixed phase forms at not too large energy densities, as e.g. in the scenarios 
discussed in Refs. [31, 32].

It is a pleasure to thank D. Blaschke, P. Danielewicz, Y.B. Ivanov, B.A. Li, J. Schaffner-Bielich and P. Senger for useful discussions.

\section{References}

[1] L. Bonanno, A. Drago, and A. Lavagno [arXiv:0704.3707 [hep-ph]].

[2] E. K. Heide, S. Rudaz, and P. J. Ellis, Nucl. Phys. A571 (1994) 713.

[3] G. W. Carter, P. J. Ellis, and S. Rudaz, Nucl. Phys. $\mathbf{A 6 0 3}$ (1996) 367; Nucl. Phys. $\mathbf{A 6 1 8}$ (1997) 317.

[4] G. W. Carter and P. J. Ellis, Nucl. Phys. A628 (1998) 325.

[5] P. Senger, J. Phys. G30 (2004) S1087.

[6] V. N. Russkikh and Y. B. Ivanov, Phys. Rev. C74 (2006) 034904.

[7] R. J. Furnstahl, B. D. Serot, and H.-B. Tang, Nucl. Phys. A598 (1996) 539.

[8] I. Mishustin, J. Bondorf, and M. Rho, Nucl. Phys. A555 (1993) 215.

[9] R. J. Furnstahl, H.-B. Tang, and B. D. Serot, Phys. Rev. C52 (1995) 1368.

[10] P. Papazoglou et al., Phys. Rev. C57 (1998) 2576.

[11] P. Papazoglou et al., Phys. Rev. C59 (1999) 411.

[12] P. Wang et al., Phys. Rev. C70 (2004) 015202.

[13] B. Liu et al., Phys. Rev. C65 (2002) 045201.

[14] G. F. Burgio et al., Phys. Rev. C66 (2002) 025802.

[15] H. Grigorian, D. Blaschke, and D. N. Aguilera, Phys. Rev. C69 (2004) 065802.

[16] V. D. Toneev et al., Eur. Phys. J. C32 (2003) 399.

[17] S. B. Ruster et al., [nucl-th/0602018].

[18] M. Alford and K. Rajagopal, [hep-ph/0606157].

[19] N. K. Glendenning, Phys. Rev. D46 (1992) 1274.

[20] H. Muller and B. D. Serot, Phys. Rev. C52 (1995) 2072.

[21] H. Muller, Nucl. Phys. A618 (1997) 349.

[22] D. N. Voskresensky, M. Yasuhira, and T. Tatsumi, Nucl. Phys. A723 (2003) 291.

[23] M. Di Toro et al., Nucl. Phys. A775 (2006) 102.

[24] W. Cassing, E. L. Bratkovskaya, and S. Juchem, Nucl. Phys. A674 (2000) 249.

[25] P. Danielewicz, R. Lacey, and W. G. Lynch, Science 298 (2002) 1592.

[26] L. Ahle et al. (E-802), Phys. Rev. C60 (1999) 044904.

[27] C. M. Hung and E. V. Shuryak, Phys. Rev. Lett. 75 (1995) 4003.

[28] H. Stoecker et al., J. Phys. G31 (2005) S929.

[29] H. Stoecker, Nucl. Phys. A750 (2005) 121.

[30] L. P. Csernai and D. Röhrich, Phys. Lett. B458 (1999) 454.

[31] I.C. Arsene et al., Phys. Rev. C75 (2007) 034902.

[32] T. Klahn et al., [nucl-th/0609067]. 